\title{
Use of whole gut perfusion to investigate gastrointestinal blood loss in patients with iron deficiency anaemia
}

\author{
A Ferguson, W G Brydon, H Brian, A Williams, M J Mackie
}

\begin{abstract}
Iron deficiency anaemia may be due to occult bleeding into the gut. However, although clinical investigations may show a high frequency of gastrointestinal tract disease in these patients, the cause-effect relationship between the lesions detected and anaemia remain uncertain. This study aimed to establish whether lesions detected by endoscopy or imaging of the gastrointestinal tract in patients with unexplained iron deficiency anaemia are bleeding continuously. Routine clinical tests were performed in 42 patients with unexplained iron deficiency anaemia referred to this unit. Whole gut lavage and assay of haemoglobin in the gut perfusate were also performed. The main outcome measures were clinical diagnoses (by imaging and endoscopy of the upper gastrointestinal tract and colon); the concentration of haemoglobin in whole gut lavage fluid; and the calculated gastrointestinal blood loss per day. There were 73 clinical, dietary, or iatrogenic factors of possible aetiological importance in the 42 patients - poor diet (10), gross gastrointestinal abnormality (34 in 28 patients), malabsorption (14), coagulation problems (6), and NSAID use (9). The gut lavage test showed, however, that at the time the test was performed, only eight patients were losing more than $2 \mathrm{ml}$ blood daily into the gut, including all four with colonic cancer, one with diffuse gastric vascular ectasia, and one with severe ulcerative oesophagitis. It is concluded that occult gastrointestinal bleeding sufficient to cause anaemia was evident in only $19 \%$ of 42 patients. There was a high frequency of other potential causes of iron deficiency in the remainder, suggesting that most of the gastrointestinal diseases and lesions detected in them were probably coincidental. Factors other than blood loss should be considered and treated in patients referred for anaemia assessment. (Gut 1996; 38: 120-124)
\end{abstract}

Keywords: occult gastrointestinal bleeding, colon cancer detection, whole gut perfusion, dietary iron deficiency, iron malabsorption.

Iron deficiency anaemia may be the only clinical manifestation of ulcerative oesophagitis, benign or malignant gastric ulcer, duodenal ulcer, large benign colonic polyps, or colonic cancer. Thus, it is normal clinical practice to examine the upper and lower gastrointestinal tract by $x$ ray or endoscopy, or both, in patients with unexplained iron deficiency. High detection rates for lesions capable of causing blood loss are reported - for example, $57 \%, 60 \%$, and $70 \%$ respectively in recent series from the USA, ${ }^{1}$ Australia, ${ }^{2}$ and England. ${ }^{3}$ Accurate measurements of the amounts of blood lost into the gut in these patients have not been performed, however, and so the cause-effect relationships between the lesions detected and the anaemia remain uncertain.

We have recently reported that when peroral gut lavage with a non-absorbable fluid is used for bowel cleansing, the clear fluid passed per rectum at the end of the procedure (whole gut lavage fluid, WGLF) is essentially a gut perfusate. ${ }^{4}$ For research purposes the lavage is supervised by an experienced nurse with a defined protocol for fluid ingestion, to give a gut perfusion rate of around $20 \mathrm{ml}$ per minute. Blood loss during the test, from any level of the gastrointestinal tract, can be detected and measured by assay of haemoglobin ( $\mathrm{Hb}$ ) in WGLF. ${ }^{5}$ We have used this new test in a series of patients with iron deficiency anaemia in order to assess which of the gastrointestinal lesions detected are chronically and continuously bleeding, and which are probably not doing so.

\section{Patients and methods}

\section{PATIENTS}

Forty two patients referred to gastrointestinal physicians with unexplained iron deficiency anaemia were investigated over an 18 month period. Twenty six were women aged between 40 and 85 years (median 66 ) and 16 were men aged between 43 and 86 years (median 72 ). Entry criteria were a low blood $\mathrm{Hb}$ concentration ( $<130 \mathrm{~g} / 1$ in men, $<115 \mathrm{~g} / 1$ in women) together with two of the following: low mean cell volume $(<76 \mathrm{f})$, low serum ferritin $(<10$ $\mu \mathrm{g} / \mathrm{l})$, low serum iron $(<14 \mu \mathrm{mol} / \mathrm{l})$, and reticulocyte response with rise in $\mathrm{Hb}$ after oral iron treatment.

In order to establish reference values for the WGLF Hb concentration, assays were performed in WGLF from 22 healthy volunteers and 15 patients with simple constipation or trivial, functional gastrointestinal symptoms; there were 23 men and 14 women, median age 32 years, range $19-86$.

Reproducibility of the technique was 
assessed indirectly, by examining results of WGLF Hb measurements in pairs of samples which had been collected from 40 patients or healthy volunteers in the course of other research projects, or as part of their routine clinical care, including 10 pairs of specimens from patients with active inflammatory bowel disease, before and after initiation of treatment.

\section{CLINICAL ASSESSMENT}

As part of the initial clinical interview and examination, we recorded not only gastrointestinal symptoms and clinical signs but also the quality of the diet and current or recent use of NSAIDs, aspirin, or anticoagulants.

Upper gastrointestinal endoscopy, rigid sigmoidoscopy, and barium enema or colonoscopy, or both, were performed in 40 patients. In two patients, when the first endoscopy examination showed a carcinoma (one gastric, one colonic) other booked investigations were cancelled. These patients have not been excluded from the series since examination of the gastrointestinal tract was completed at laparotomy. Although the planned protocol included endoscopic duodenal biopsies for the diagnosis of coeliac disease, these were, in fact, obtained from only 25 patients, and a further three had a Watson capsule biopsy of the jejunum.

\section{GUT LAVAGE PROCEDURE}

Gut lavage was performed as preparation for barium enema or colonoscopy examination. Supervised by a research nurse, patients drank isotonic lavage fluid (Klean-Prep, Norgine $\mathrm{Ltd}, \mathrm{UK})$, at a rate of one glass $(200 \mathrm{ml})$ every 10-15 minutes, until clear fluid was being passed per rectum. A sample was collected and stored at $-70^{\circ} \mathrm{C}$.

\section{ASSAY OF HAEMOGLOBIN AND CALCULATION OF} BLOOD LOSS

Haemoglobin in WGLF was assayed by the Hemoquant technique, which measures bacterially degraded as well as intact $\mathrm{Hb}^{6}{ }^{6}$ The blood $\mathrm{Hb}$ concentration was measured in a sample taken on the day of lavage and gastrointestinal blood loss per day was calculated as:

$\frac{\text { WGLF Hb concentration }(\mu \mathrm{g} / \mathrm{ml}) \times 28 \cdot 8}{\text { Blood } \mathrm{Hb} \text { concentration }(\mathrm{g} / \mathrm{l})}=\mathrm{ml}$ blood lost $/ \mathrm{d}$
$($ this assumes a perfusion rate of 20
$\mathrm{ml} / \mathrm{min}=28.8 \mathrm{l} / \mathrm{d})$.

\section{Results}

CLINICAL FEATURES

Initial $\mathrm{Hb}$ values in the 16 men ranged from $61-119 \mathrm{~g} / 1$ (mean $90 \mathrm{~g} / \mathrm{l}$ ), and in the 26 women they ranged from 45-114 g/l (mean $85 \mathrm{~g} / \mathrm{l}$ ). Many patients had symptoms of anaemia such as tiredness, lethargy, and breathlessness but few had significant gastrointestinal symptoms.

One woman developed dysphagia while being investigated; five patients reported dyspepsia, three heartburn, one constipation, and two diarrhoea.

There were 10 patients whose diet was clearly deficient in iron-containing foods (as judged at clinical interview by one of us (AF, AW, or MM); formal dietary assessment was not performed. Nine patients had been taking NSAIDs when the anaemia was diagnosed but at the time of their gastrointestinal investigations the drugs had been withdrawn from eight of the nine. Four patients had been on long term anticoagulant therapy, which had been stopped in one case. There was a further patient with a bleeding diathesis due to idiopathic thrombocytopenic purpura and one woman with alcoholic liver disease also had abnormal coagulation.

\section{MALABSORPTION}

Twelve patients had a gastric abnormality likely to lead to iron malabsorption. Seven of the 12 had had a partial gastrectomy for peptic ulcer disease many years previously and the other five had atrophic gastritis.

Small bowel biopsy tissue from one woman with no gastrointestinal symptoms and a diet deficient in iron showed pathology typical of coeliac disease. Coeliac disease was probable in one other patient with an oesophageal carcinoma. She had old rickets and gave a history of macrocytic anaemia with a normal Schilling test 25 years ago; unfortunately the endoscopist had not taken a duodenal biopsy but ELISAs were positive for serum IgA and WGLF IgA antibodies to gliadin. ${ }^{7}$

\section{OTHER GASTROINTESTINAL DISEASES}

\section{DETECTED}

Standard $x$ ray and endoscopic investigations produced many positive results, including six carcinomas (Table I). Indeed there were only seven patients in whom no lesion was found:

TABLE I Gastrointestinal diseases and other factors of possible relevance to iron deficiency anaemia in 42 patients

\section{No of patients}

Malabsorption:

Atrophic gastritis

Partial gastrectomy without gastritis

Partial gastrectomy with gastritis

Partial gastrectom

Coeliac-definite
Coeliac-probable

Other gastrointestinal diseases:

Oesophagitis mild

Oesophagitis severe ulcerative

Carcinoma oesophagus

Hiatus hernia

Acute gastritis

Gastric vascular ectasia

Gastric carcinoma

Duodenal ulcer

Crohn's (ileum)

Colon cancer

Colon cancer
Benign colonic polyps

Benign colonic polyps
Diverticular disease

Abnormal coagulation:

On anticoagulants

Previously anticoagulated now stopped

Idiopathic thrombocytopenic purpura

Alcoholic liver disease

Drugs:

Previously taking NSAID

Current on NSAID

Poor diet:

Iron deficient diet 


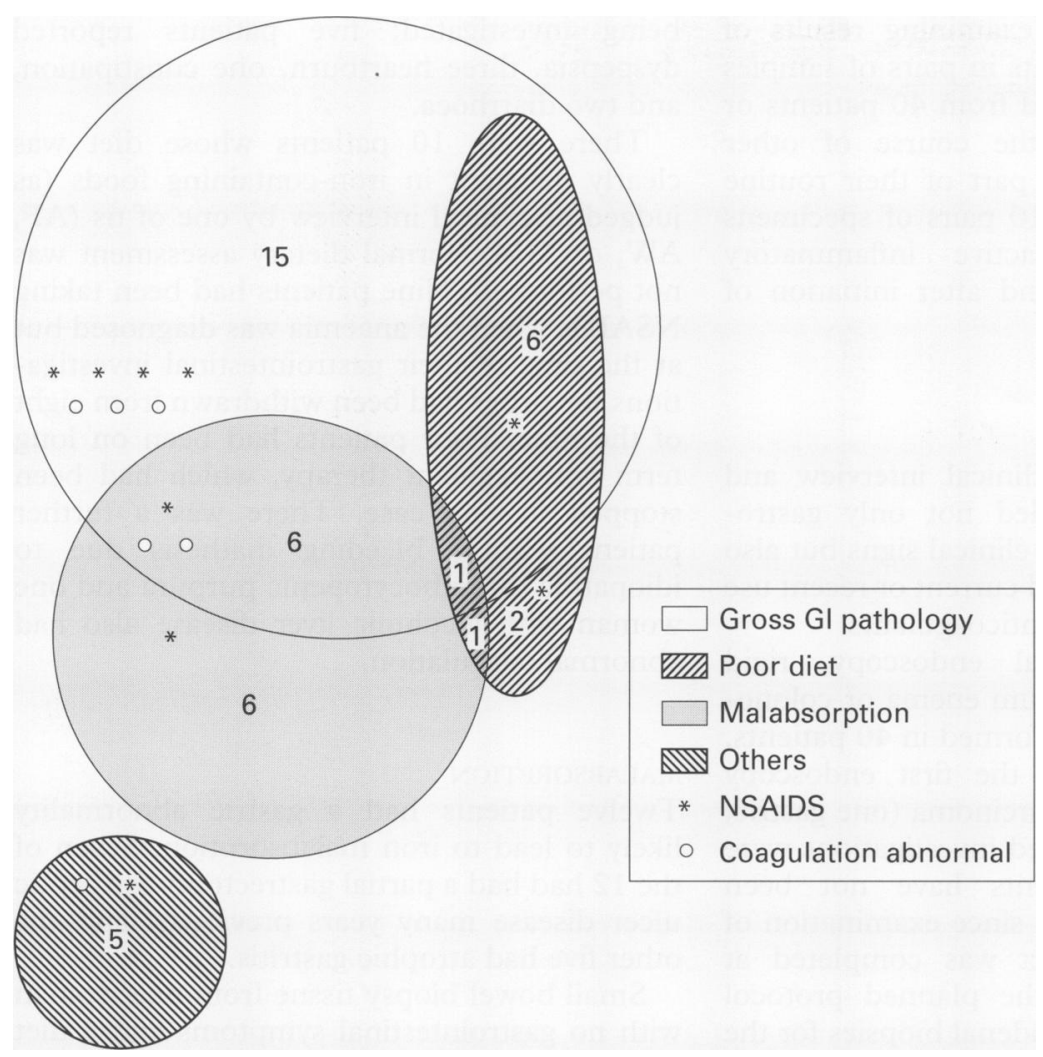

Figure 1: Presence of gastrointestinal (GI) diseases and of other aetiological factors in 42 patients with iron deficiency anaemia. In 28 patients, one or more gastrointestinal disease was detected by imaging or endoscopy, or both; in 10 the diet was judged by an experienced clinician to be deficient in iron; and 14 patients had diseases associated with iron malabsorption. As shown, there was significant overlap between these groups and with the presence of other relevant factors such as use of non-steroidal anti-inflammatory drugs (NSAIDs) or coagulation abnormality.

two of these had iron deficient diets. one had alcoholic liver disease, one had previously taken NSAIDs, leaving only three of the 42 patients for whom we had completely negative findings (Fig 1).

\section{MEASUREMENT OF GASTROINTESTINAL BLEEDING}

Values for WGLF $\mathrm{Hb}$ in the 37 volunteers and patients with a normal gastrointestinal tract, ranged from $1 \cdot 0-5 \cdot 4 \mu \mathrm{g} / \mathrm{ml}$ (mean $2 \cdot 50$, SD $1 \cdot 13)$. We have therefore set the reference range for this measurement as $1-5 \mu \mathrm{g} / \mathrm{ml}$.

Thirty sets of duplicate samples were from individuals with normal values for WGLF $\mathrm{Hb}$ at the first test. In all cases the value for the second sample was also normal; mean

TABLE II Concentrations of IgG (reflecting disease activity) and of haemoglobin, in whole gut lavage fluid (WGLF) from a series of patients with inflammatory bowel disease who had gut lavage performed twice within a four week period

\begin{tabular}{cccccc}
\hline & \multicolumn{2}{l}{$I g G(\mu g / m l)$ in $W G L F$} & & \multicolumn{2}{c}{$H(\mu g / m l)$ in $W G L F$} \\
\cline { 2 - 3 } \cline { 5 - 6 } & Relapse & $\begin{array}{c}\text { On } \\
\text { treatment }\end{array}$ & & Relapse & $\begin{array}{l}\text { On } \\
\text { treatment }\end{array}$ \\
\hline Crohn's & 55 & 24 & & 10 & 5 \\
disease & 93 & 60 & & 22 & 9 \\
& 144 & 82 & & 162 & 145 \\
& 137 & 54 & & 58 & 14 \\
& 27 & 9 & & 12 & 9 \\
& 216 & 41 & & 15 & 9 \\
Ulcerative & 44 & 34 & & 9 & 8 \\
colitis & 254 & 11 & & 14 & 4 \\
& 188 & 2 & & 151 & 25 \\
& & & & & \\
\hline
\end{tabular}

difference in duplicate values was $0.96 \mu \mathrm{g} / \mathrm{ml}$ (range 0-3). There were 10 pairs of specimens from patients with inflammatory bowel disease, one sample collected in relapse, and one after a variable clinical improvement on treatment. A shown in Table II, concentrations of IgG and of $\mathrm{Hb}$ in WGLF parallelled disease activity: $\mathrm{Hb}$ concentrations were lower in the specimen collected after treatment, but in most cases the values were still above the reference range.

Twenty five of the 42 patients with iron deficiency had values for WGLF Hb within the normal range. There were a further seven patients with marginally raised concentrations (6-8 $\mu \mathrm{g} / \mathrm{ml}$, calculated daily gastrointestinal blood losses $1 \cdot 2-1 \cdot 7 \mathrm{ml} / \mathrm{d}$ ), including two patients with malignant disease (gastric cancer, $6 \mu \mathrm{g} / \mathrm{ml}, 1.2 \mathrm{ml} / \mathrm{d}$, oesophageal cancer $6 \mu \mathrm{g} / \mathrm{ml}$, $1.3 \mathrm{ml} / \mathrm{d}$ ). Two patients had WGLF $\mathrm{Hb}$ concentrations of 10 and $12 \mu \mathrm{g} / \mathrm{ml}$, with calculated blood losses of borderline clinical significance at 2.0 and $2.1 \mathrm{ml} / \mathrm{d}$, and there were only eight patients with unequivocally high values (taken as WGLF $\mathrm{Hb}>10 \mu \mathrm{g} / \mathrm{ml}$, and calculated blood loss $>2 \mathrm{ml} / \mathrm{d}$ during the lavage procedure) (Fig 2). This group included all four patients with colon cancer (blood losses $5-132 \mathrm{ml} / \mathrm{d}$ ).

\section{Discussion}

In view of the very high rates of detection of gastrointestinal diseases in anaemic patients, it has been entirely reasonable to assume that chronic blood loss from the gut is the commonest cause of iron deficiency anaemia in men and in postmenopausal women. ${ }^{8}$ Lack of correlation with positive faecal occult blood test has generally been attributed to deficiencies in the guaiac based faecal occult blood methods, which are relatively insensitive and preferentially detect blood loss from the distal gastrointestinal tract. A further factor is that bleeding may occur in the form of multiple intermittent episodes, rather than as a continuous ooze.

The accurate measurement of bleeding into the gut requires a suitable assay for $\mathrm{Hb}$ or some other component of red blood cells, applied to a specimen representative of gut luminal contents - ideally a complete five or seven day faecal collection or several separate faecal samples. Problems associated with tests on faeces include not only the resistance to collection and handling of faecal specimens by patients, nurses, and laboratory staff, but also the potential interference in analyses by residues of meat and other dietary constituents. Patient-related variables such as intestinal transit time and faecal bulk are also relevant. The Hemoquant method is a highly sensitive analytical technique, which measures both intact and bacterially metabolised haemoglobin ${ }^{7}$; when this is used to test a timed specimen of faeces collected after a period on a meat free diet, it can detect and quantify blood loss from the proximal or distal gut. We have previously reported that this method can also readily be used with WGLF. ${ }^{5}$ Use of gut 


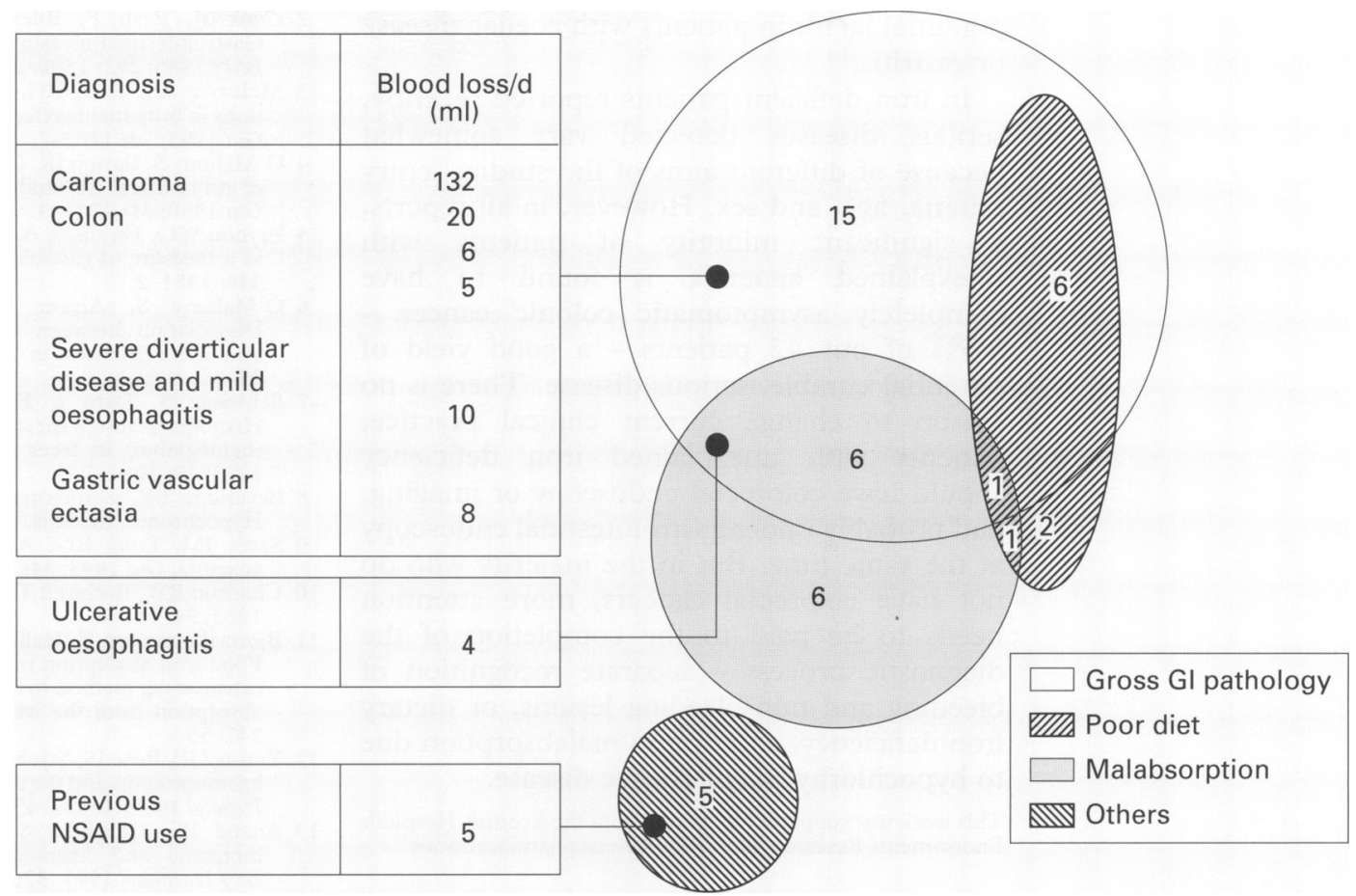

Figure 2: Distribution (within the classification shown in Figure 1) and diagnosis, for the eight patients in whom gastrointestinal blood loss (based on the whole gut lavage fluid test) was estimated to be more than $2 \mathrm{ml}$ daily.

perfusion fluid as an assay material overcomes the need for dietary restrictions and unpleasant faecal collections, and specimens can be obtained when peroral gut lavage is being used to cleanse the bowel before colonic investigations. The reference range for WGLF $\mathrm{Hb}$ concentrations, $1-5 \mu \mathrm{g} / \mathrm{ml}$, equates to daily blood losses of around $0 \cdot 2-1 \mathrm{ml} / \mathrm{d}$. The main theoretical disadvantage of this new test of gastrointestinal bleeding is that the sampling time is limited to a few hours; further experience, and comparisons between WGLF and five day faecal collections, should show how frequently occult bleeding is discontinuous, and in what time frame.

Review of the results of WGLF $\mathrm{Hb}$ measurements highlights the complexity of possible aetiologies in the 42 iron deficient patients studied. Altogether 73 clinical, dietary, or iatrogenic factors were recorded poor diet (10), gross gastrointestinal abnormality (34 in 28 patients), malabsorption (14), coagulation problems (6), and NSAID use (9). However, out test of gastrointestinal bleeding detected only eight patients who were losing more than $2 \mathrm{ml}$ blood daily into the gut, including all six patients with lesions generally recognised to be important causes of occult bleeding - four with colonic cancer, one with diffuse gastric vascular ectasia, and one with severe, ulcerative oesophagitis.

There are several possible explanations for the findings. In some patients with low or normal values for WGLF $\mathrm{Hb}$, gastrointestinal bleeding may be intermittent rather than a continuous ooze, as discussed above. The lesion responsible for blood loss might have healed in the interval between diagnosis of anaemia and investigation. This may well have been the case in NSAID treated patients, eight of whom had the drugs stopped as soon as anaemia was recognised. It seems likely, however, that in many instances, the gastrointestinal lesions detected by standard investigations were simply not bleeding, and the anaemia was the result of some other cause. In the patients with calculated daily blood loss of less than $2 \mathrm{ml} / \mathrm{d}$, based on the WGLF test, there was a high frequency of other conditions which might lead to iron deficiency, such as poor diet or malabsorption. Regrettably, in developing the protocol for the study we did not arrange a formal assessment of dietary iron intake, but a general appraisal of the diet was made by an experienced and nutritionally aware clinician. Nevertheless, this is an example of how the emphasis which we and others have placed on the development of better tests for gastrointestinal bleeding leads to relative neglect of these other clinically important features.

Absorption and bioavailability of dietary iron, and iron malabsorption, are partly interrelated. The amount of inorganic iron which is absorbed is greatly influenced by the nature of other foods taken at the same time. ${ }^{10} \mathrm{~A}$ much higher proportion of haem iron than inorganic iron is absorbed, ${ }^{11} 12$ and malabsorption syndromes may affect one and not the other - for example, in coeliac disease there is malabsorption of ferrous but not of haem iron. ${ }^{13}$ Optimal inorganic iron absorption requires healthy gastric and small bowel mucosae, and so will be compromised after gastric surgery, and probably also in patients with atrophic gastritis and hypochlorhydria. There were seven (17\%) patients in our series who had had a partial gastrectomy many years before. Coeliac disease may be expressed as a single nutrient deficiency, and some form of small bowel biopsy should be included in the investigation of all iron deficient patients. Free iron loss into the gut in association with high rates of epithelial cell proliferation and loss, is another 
potential factor in patients with coeliac disease or gastritis. ${ }^{14}$

In iron deficient patients reported recently, serious diseases detected vary somewhat because of different aims of the studies, entry criteria, age, and sex. However, in all reports, a significant minority of patients with unexplained anaemia is found to have completely asymptomatic colonic cancer $9.5 \%$ of our 42 patients - a good yield of potential curable, serious disease. There is no reason to change current clinical practice; patients with unexplained iron deficiency should have colorectal endoscopy or imaging, and probably upper gastrointestinal endoscopy at the same time. But in the majority who do not have colorectal cancers, more attention needs to be paid to the completion of the diagnostic process - separate recognition of bleeding and non-bleeding lesions, or dietary iron deficiency, and of iron malabsorption due to hypochlorhydria or coeliac disease.

This work was supported by grants from the Scottish Hospitals Endowments Research Trust, and Fisons pharmaceuticals.

1 Rockey DC, Cello JP. Evaluation of the gastrointestinal tract in patients with iron-deficiency anemia. $N$ Engl $\mathcal{f}$ Med 1993; 329: 1691-5.
2 Cook IJ, Pavli P, Riley JW, Goulston KJ, Dent O. Gastrointestinal investigation of iron deficiency anaemia. BMF 1986; 292: 1380-2.

3 McIntyre AS, Long RG. Prospective survey of investigations in outpatients referred with iron deficiency anaemia. Gut 1993; 34: 1102-7.

4 O'Mahony S, Barton JR, Crichton S, Ferguson A. Appraisal of gut lavage in the study of intestinal humoral immunity. Gut 1990; 31: 1341-4.

5 Brydon WG, Ferguson A. Haemoglobin in gut lavage fluid as a measure of gastrointestinal blood loss. Lancet 1992; 340: 1381-2.

6 O'Mahony S, Arranz E, Barton JR, Ferguson A. Dissociation between systemic and mucosal humoral immune responses in coeliac disease. Gut 1991; 32: 29-35.

7 Schwartz S, Dahl J, Ellefson $M$, Ahlquist DA. The Hemoquant test; a specific and quantitative assay of heme (hemoglobin) in feces and other materials. Clin Chem 1983; 29: 2061-7.

8 Beveridge BR, Bannerman RM, Evanson JM, Witts LJ. Hypochromic anaemia. $Q \mathcal{F}$ Med 1965 ; 34: 145-61.

9 Sayer JM, Long RG. A perspective on iron deficiency Sayer JM, Long RG. A perspectiv
anaemia. Gut 1993; 34: 1297-9.

10 Charlton RW, Bothwell TH. Iron absorption. Ann Rev Med 1983; 34: 55-68.

11 Bjorn-Rasmussen E, Hallberg L, Isaksson B, Arvidsson B. Food iron absorption in man. Applications of the 2 pool extrinsic tag method to measure haem and non haem iron absorption from the whole gut. $f$ Clin Invest 1974; 53: 247-55.

12 Young GP, Rose IS, StJohn DJB. Haem in the gut I. Fate of haemoproteins and the absorption of haem. $\mathcal{F}$ Gastroenterol Hepatol 1989; 4: 537-45.

13 Anand BS, Callender ST, Warner GT. Absorption of inorganic and haemoglobin iron in coeliac disease. Br f Haematol 1977; 37: 409-14.

14 Sutton DR, Stewart JS, McL Baird I, Coghill NF. Free iron loss in atrophic gastritis, post gastrectomy states, and adult coeliac disease. Lancet 1970; i: 387-90. 